\title{
A fault-tolerant hexagonal systolic array
}

\author{
C. Aykanat * \\ Department of Computer and Information Science, Bilkent Liniersity, P.K. 8, 06572 Maltepe, Arkara, Turkey
}

\section{F. Özgüner}

Department of Elecrical Engineering, The Ohio State University, Columbus, OH 43210, USA

Communicated by F.B. Schneider

Received 11 October 1989

Revised 11 March 1992

Kcywords: Fault tolerance, systolic array, error detecting

\section{Introduction}

Systolic array structures have been proposed as a cost effective means of achieving high performance computing for a wide range of compute bound applications such as real-time signal and image processing and matrix computations [6,7]. Fault tolerance is an important issue in these architectures to ensure the correctness of computations. Time redundancy techniques for fault detection and correction in systolic arrays are not desirable due to real-time constraints. Thus, hardwate redundancy would be the most efficicnt alternative for fault tolerance. Careful analysis of some bidirectional systolic algorithms reveals the fact that a substantial number of PE's remain idle to provide correct timing and sequencing of data operands. This feature has been used to perform computations in duplicate for error detection in [4] for lincar arrays and in [3] for hexagonal arrays. In this paper, a concurrent error detecting systolic design and algorithm will be presented for band matrix multiplication on a hexagonal

Correspondence to: F. Özgüner, Department of Electrical Engineering, The Ohio State University, 205 Dreese Laboratory, 2015 Neil Avenue, Columbus, OH 43210, USA. Email: ozguner@bambi.eng.ohio-state.edu.

* Email: aykanat@utrblin.bitnet. systolic array, that uses fewer comparators than Choi's design [3]. An error correcting design is also presented that performs computations in triplicate by utilizing the property that in any row or column of the systolic array, out of every three consccutive PE's, only one is active at any given time.

Band matrix multiplication represents the inner loop of many real-time compute bound tasks [5]. The two-dimensional systolic network of hexagonally connected processors shown in Fig. 1 has been proposed by Kung and Leiserson [7] for the multiplication of band matrices. The original systolic algorithm proposed by Kung and Leiserson was refined by Huang and Abraham in [5] to minimize turnaround time and maximize processor utilization. However, the increase in the performance obtained by the Huang-Abraham systolic algorithm is achieved by trading off the relaxed $1 / O$ bandwidth of the Kung Leiserson algorithm. Thus, the Kung-Leiserson systolic algorithm is best suited for the multiplication of wide-banded matrices, whereas the HuangAbraham algorithm is best suited for narrowbanded matrices which require fewer data transfers from the host computer [2]. In [5] a design technique for an crror detecting hexagonal array is also given that is based on encoding the input matrices and checking the encoded output ma- 